
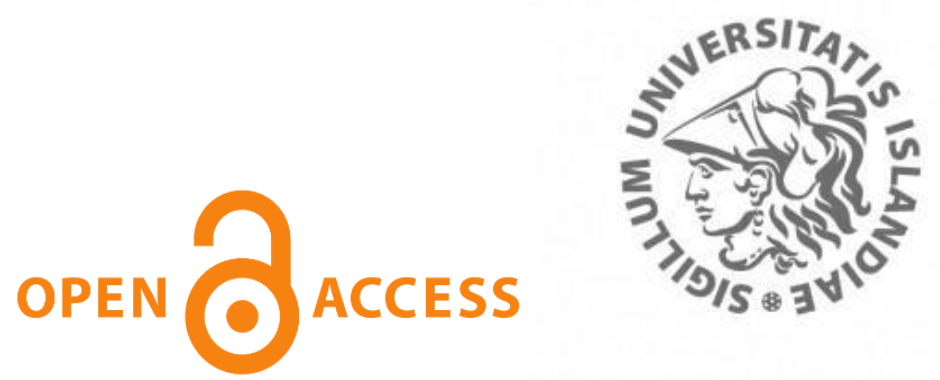

O. Opin vísindi

This is not the published version of the article / Petta er ekki útgefna útgáfa greinarinnar

Author(s)/Höf.: $\quad$ Ragnar Bjornsson, Frank Nesse and Serena DeBeer

Title/Titill: $\quad$ Revisiting the Mössbauer Isomer Shifts of the FeMoco Cluster of Nitrogenase and the Cofactor Charge

Year/Útgáfuár: $\quad 2017$

Version/Útgáfa: $\quad$ Post-print / lokagerđ höfundar

Please cite the original version:

Vinsamlega vísið til útgefnu greinarinnar:

Bjornsson, R., Neese, F., \& DeBeer, S. (2017). Revisiting the Mössbauer Isomer Shifts of the FeMoco Cluster of Nitrogenase and the Cofactor Charge. Inorganic Chemistry, 56(3), 1470-1477. doi:10.1021/acs.inorgchem.6b02540

Rights/Réttur: $\quad$ Copyright @ 2017American Chemical Society 


\title{
Revisiting the Mössbauer isomer shifts of the FeMoco cluster of nitrogenase and the cofactor charge
}

\author{
Ragnar Bjornsson, ${ }^{*, \dagger, \ddagger}$ Frank Neese, ${ }^{\dagger}$ and Serena DeBeer ${ }^{*, \dagger, \uparrow}$ \\ †Max Planck Institute for Chemical Energy Conversion, Stiftstr. 34-36, 45470 Mülheim an \\ der Ruhr, Germany \\ $\ddagger$ Science Institute, University of Iceland, Dunhagi 3, 107 Reykjavik, Iceland \\ \Department of Chemistry and Chemical Biology, Cornell University, Ithaca, NY 14853 , \\ United States \\ E-mail: ragnar.bjornsson@gmail.com; serena.debeer@cec.mpg.de
}

\begin{abstract}
Despite decades of research, the structure-activity relationship of nitrogenase is still not understood. Only recently was the full molecular structure of the FeMo cofactor (FeMoco) revealed but the charge and metal oxidation states of FeMoco have been controversial. With the recent identification of the interstitial atom as a carbide and the more recent revised oxidation state assignment of the molybdenum atom as Mo(III), here we revisit the Mössbauer properties of FeMoco. By a detailed error analysis of DFT-computed isomer shifts and computing isomer shifts relative to the P-cluster, we find that only the charge of $\left[\mathrm{MoFe}_{7} \mathrm{~S}_{9} \mathrm{C}\right]^{1-}$ fits the experimental data. In view of the recent Mo(III) identification, the charge of
\end{abstract}


$\left[\mathrm{MoFe}_{7} \mathrm{~S}_{9} \mathrm{C}\right]^{1-}$ corresponds to a formal oxidation state assignment of $\mathrm{Mo}(\mathrm{III}) 3 \mathrm{Fe}(\mathrm{II}) 4 \mathrm{Fe}(\mathrm{III})$, although due to spin delocalization, the physical oxidation state distribution might also be interpreted as $\mathrm{Mo}(\mathrm{III}) 1 \mathrm{Fe}(\mathrm{II}) 4 \mathrm{Fe}(2.5) 2 \mathrm{Fe}(\mathrm{III})$, according to a localized orbital analysis of the $\mathrm{M}_{\mathrm{S}}=3 / 2$ broken symmetry solution. These results can be reconciled with the recent spatially resolved anomalous dispersion (SpReAD) study by Einsle et al. that suggests the $\mathrm{Mo}(\mathrm{III}) 3 \mathrm{Fe}(\mathrm{II}) 4 \mathrm{Fe}(\mathrm{III})$ distribution, if some spin localization (either through interactions with the protein environment or through vibronic coupling) were to take place.

\section{Introduction}

Molybdenum-dependent nitrogenase is a complex enzyme that catalyzes the formation of ammonia through the electrochemical reduction of molecular nitrogen at ambient temperature and pressure, utilizing eight electrons, eight protons and $16 \mathrm{MgATP}$ molecules. Unraveling the structural and mechanistic details of how nature activates the strong bond of dinitrogen is of fundamental importance and this knowledge could ultimately aid in the design of electrocatalysts for $\mathrm{N}_{2}$ reduction. The molybdenum-iron component ( $\mathrm{MoFe}$ ) of nitrogenase has two types of complex metal clusters, the "P-cluster" and the FeMo cofactor (FeMoco). The P-cluster appears to serve as an electron transfer site, while FeMoco is generally agreed to be the site of dinitrogen reduction. The FeMo cofactor consists of 7 irons, 1 molybdenum, 9 sulfides and an interstitial light atom that was recently identified as carbon by X-ray Emission Spectroscopy(XES), ${ }^{1}$ Electron Spin Echo Envelope Modulation (ESEEM) and high resolution crystallography. ${ }^{2}$ With the identity of the interstitial atom clear, the basic molecular structure of the cofactor can at last be considered complete. However, questions remain about the resting form of the enzyme, namely the total charge of the cofactor, metal oxidation states and the electronic structure. This information is essential for any informed discussion of the molecular level mechanism of dinitrogen reduction. In the present 
study, we focus on the cofactor charge, a question directly related to the question of metal oxidation states in the cofactor.

The charge of FeMoco was determined to be negative according to electrophoresis, ${ }^{3}$ DEAE binding studies ${ }^{4}$ and counterion chromatography. ${ }^{5}$ The first Mössbauer studies of the cofactor were inconclusive regarding the number of Fe atoms and Fe oxidation states. ${ }^{6-8}$ Following the first crystal structure of MoFe protein in $1992,{ }^{9}$ but prior to the interstitial atom discovery in $2002,{ }^{10}$ several studies proposed charge and oxidation state assignments of FeMoco. In our recent paper, ${ }^{11}$ we gave a brief history of the oxidation state assignment of molybdenum, highlighting how prior to our study it had almost exclusively been assigned as Mo(IV), since ${ }^{95}$ Mo ENDOR studies from the 1980s. ${ }^{12-14}$ This assignment has, as it turns out, directly affected many Fe oxidation state assignment studies, as the total d-electron count of the cofactor is chosen to be consistent with the experimental spin state of $\mathrm{S}=3 / 2 .{ }^{15}$ The 1997 study by Hoffman et al. ${ }^{16}$ employed ${ }^{57}$ Fe Electron Nuclear Double Resonance (ENDOR) spectroscopy to assign Fe oxidation states, assumed Mo(IV) on the basis of previous ${ }^{95} \mathrm{Mo}$ ENDOR results and suggested a Mo(IV)6Fe(II)1Fe(III) assignment (a d-electron count of 43). The electronic structure was suggested to consist of a delocalized $\mathrm{Fe}(2.5)-\mathrm{Fe}(2.5)$ pair and $5 \mathrm{Fe}(\mathrm{II})$. A magnetic Mössbauer spectroscopy study by Münck et al. ${ }^{17}$ from 2000 was able to identify all seven irons in FeMoco, giving both isomer shifts, quadrupole splittings and hyperfine coupling parameters, see Table 1 . The molybdenum atom was assumed to be $\mathrm{Mo}(\mathrm{IV})$ on the basis of the ENDOR results and the authors assigned the iron atoms as $4 \mathrm{Fe}(\mathrm{II})$ and $3 \mathrm{Fe}(\mathrm{III})$. As this study was performed prior to the interstitial atom discovery, it seems that this incomplete structure (no interstitial atom present) as well as the assumption of $\mathrm{Mo}(\mathrm{IV})$ directly influenced the analysis. In later work by Vrajmasu et al. ${ }^{18}$ DFT calculations of isomer shifts were performed, however, the molybdenum atom was not included in the DFT calculations.

The computational study by Szilagyi et al. ${ }^{19}$ was the most recent computational study 
to discuss the cofactor charge question in detail. Many different computational models of FeMoco with different charge and interstitial atoms were compared to experimental data on spin states, geometries, Mössbauer isomer shifts and iron hyperfine coupling constants. The computational model that showed the best fit to experimental data was found to contain carbon (as is now confidently known), but the oxidation state assignment was curiously found to be $2 \mathrm{Fe}(\mathrm{II}) 5 \mathrm{Fe}(\mathrm{III}) \mathrm{Mo}(\mathrm{IV})$ (d-electron count of 39), a more oxidized FeMoco model than previous proposals. In particular, the comparison of computed and experimental Mössbauer isomer shifts suggested the more oxidized cofactor to be the correct charge state.

X-ray spectroscopy has played an important role in recent years in uncovering the molecular and electronic structure aspects of $\mathrm{FeMoco}^{20}$ and in a recent Mo HERFD-XAS (high energy resolution fluorescence detection X-ray absorption spectroscopy) study ${ }^{11}$ of MoFe protein, we gave a new oxidation state assignment of the molybdenum, being a Mo(III) ion, rather than $\mathrm{Mo}(\mathrm{IV})$, based on the experimental Mo K-edges of the protein and model compounds and supported by DFT calculations. Using the combined HERFD-XAS/DFT approach, we attempted a charge assignment as well, but found that the protocol was not sensitive enough to allow for a clear assignment. The Mo(III) assignment of FeMoco requires a reevaluation of the Fe oxidation state assignments commonly found in the literature (while the charge possibilities remain the same). In order to be consistent with an $S=3 / 2$ ground state spin (as known from EPR spectroscopy ${ }^{15}$ ), the previously proposed FeMoco oxidation state assignments have to be reassigned, as shown in Figure 1. While metal oxidation state assignment remains crucial to understanding FeMoco, we prefer discussing possible charge states of resting state FeMoco in terms of total charge of the cofactor, (excluding the homocitrate as well as the amino acid residues bound to the cofactor) and with sulfur and carbon taken in their usual closed-shell forms, $\mathrm{S}^{2-}$ and $\mathrm{C}^{4-}$. This allows a more unambigous discussion as there is no dependence on oxidation state interpretation or the protonation state of homocitrate. Figure 1 shows the charge states of FeMoco in terms of total charge of the 
pure cofactor and also in terms of oxidation states (with both the new Mo(III) assignment as well as the previous Mo(IV) one). Also included is the total metal d-electron count.

Very recently, Spatzal et al. ${ }^{21}$ applied spatially resolved anomalous dispersion (SpReAD) to MoFe protein and were able to acquire site-specific Fe K-edges of each Fe atom in FeMoco and P-cluster. The data reveals $3 \mathrm{Fe}$ atoms to have lower-energy edge positions (similar to P-cluster Fe atoms) and thus likely more reduced than the other 4, suggesting the oxidation assignment of $3 \mathrm{Fe}(\mathrm{II}) 4 \mathrm{Fe}(\mathrm{III}) \mathrm{Mo}(\mathrm{III})$ when combined with the identification of the molybdenum as $\mathrm{Mo}(\mathrm{III})$ and hence a charge of $\left[\mathrm{MoFe}_{7} \mathrm{~S}_{9} \mathrm{C}\right]^{1-}$.

Computational papers on FeMoco have in recent years focused on possible mechanisms for $\mathrm{N}_{2}$ reduction, many of these studies appear to assume a charge of $\left[\mathrm{MoFe}_{7} \mathrm{~S}_{9} \mathrm{C}\right]^{1-}$ for the cofactor (often the charge is described in terms of oxidation state e.g. 4Fe(II)3Fe(III)Mo(IV)). A recent mechanistic study by Siegbahn ${ }^{22}$ agrees with the Mo(III) assignment and uses $\left[\mathrm{MoFe}_{7} \mathrm{~S}_{9} \mathrm{C}\right]^{1-}$ for the resting state, arguing that other charges give unreasonable energetics for the mechanism. However, conflict remains in the literature, with a recent SpReAD study suggesting $\left[\mathrm{MoFe}_{7} \mathrm{~S}_{9} \mathrm{C}\right]^{1-}$ while the latest interpretation of the Mössbauer properties by Szilagyi et al. suggests $\left[\mathrm{MoFe}_{7} \mathrm{~S}_{9} \mathrm{C}\right]^{1+}$.

Having the knowledge of a complete molecular structure and a larger FeMoco active site model than used in previous studies, we now revisit the Mössbauer spectra of FeMoco. Using DFT calculations and a detailed analysis of the predicted Mössbauer isomer shifts, we demonstrate that the $\left[\mathrm{MoFe}_{7} \mathrm{~S}_{9} \mathrm{C}\right]^{1-}$ charge is in best agreement with the experimental Mössbauer data.

\section{Computational details}

All DFT computations were carried out using the ORCA program, version 3.0.3. ${ }^{23}$ Geometry optimizations of all compounds were performed at the $\mathrm{TPSSh}^{24}$ level of theory (employing 


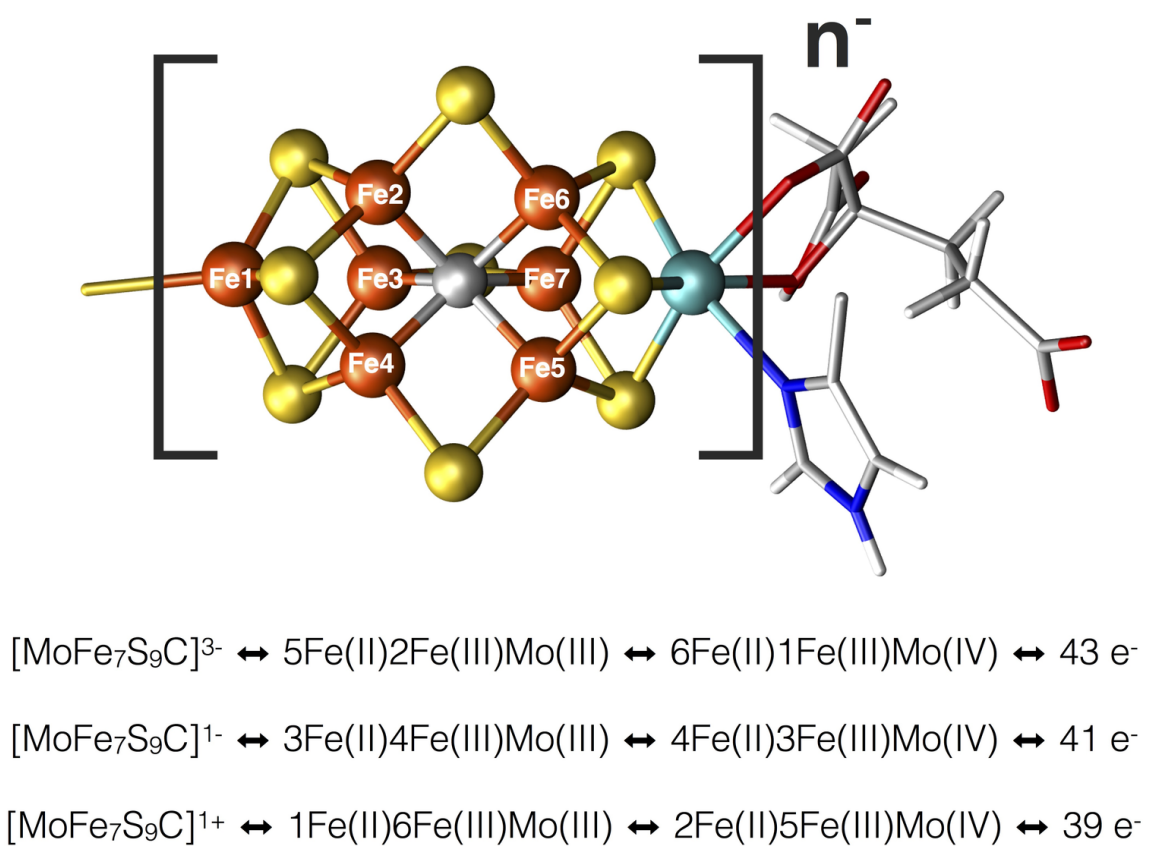

Figure 1: The relation between FeMo cofactor charge, metal oxidation states and d-electron count. The metal oxidation states are shown both using the recent $\mathrm{Mo}(\mathrm{III})$ assignment $^{11}$ and the previous $\mathrm{Mo}(\mathrm{IV})$ assignment. ${ }^{12-14}$ Only the atoms within the brackets $\left(\mathrm{MoFe}_{7} \mathrm{~S}_{9} \mathrm{C}\right)$ count towards the charge. Protonation states of homocitrate and histidine do not affect this charge definition.

the RIJCOSX approximation, ${ }^{25,26}$ ) including the ZORA scalar relativistic method ${ }^{27}$ and relativistically recontracted versions of the Karlsruhe def2-TZVP basis sets. ${ }^{28,29}$ A dielectric field was introduced using the COSMO approximation ${ }^{30}$ in order to account for bulk protein environmental effects. Single-point calculations were performed using the BP86 GGA functional, the hybrid meta-GGA TPSSh functional (10\% HF exchange) and the hybrid GGA functional B3LYP (20\% HF exchange) to obtain electron density values at the Fe nuclear positions which were used to derive the isomer shifts. A mixed basis set was used that consists of the core-polarized CP(PPP) basis set ${ }^{31,32}$ on Fe atoms and DKH-def2-TZVP 28,29 on all other atoms. Scalar relativistic effects in the Mössbauer isomer shift calculations were accounted for using the second-order Douglas-Kroll-Hess approximation. Localized orbitals were calculated using the Pipek-Mezey scheme. ${ }^{33}$ The protein-bound FeMoco cluster was 
approximated as a large 225 atom cluster previously described. ${ }^{11}$ This computational model includes part of the protein environment and crystallographically resolved water molecules from the 2011 X-ray structure by Einsle et al. and was calculated in the 3 charge states $\left(\left[\mathrm{MoFe}_{7} \mathrm{~S}_{9} \mathrm{C}\right]^{\mathrm{n}} \mathrm{n}=-3,-1,+1\right)$, currently proposed in the literature. Broken-symmetry solutions of FeMoco were found by first converging to high-spin SCF solutions $\left(\mathrm{M}_{\mathrm{S}}=33 / 2, \mathrm{M}_{\mathrm{S}}=35 / 2\right.$, $\mathrm{M}_{\mathrm{S}}=37 / 2$ for charges $\left.\mathrm{n}=-3,-1,+1\right)$ and then performing spin flips on selected atoms and converging to broken-symmetry states of a particular $\mathrm{M}_{\mathrm{S}}$ value. Both X-ray crystal structure geometries were used as well as geometries optimized at the TPSSh level on the brokensymmetry surface using appropriate constraints (all non-covalently bound protein residues were fixed at X-ray crystal structure positions except for hydrogen atoms).

Table 1: Experimental Mössbauer isomer shifts and quadrupole couplings from the magnetic Mössbauer study by Münck et al. ${ }^{17}$. Measurements performed at $4.2 \mathrm{~K}$ with isotope labelled MoFe protein. Fe site labels are from the study by Münck et al.

$\begin{array}{cccc}\text { Fe atom } & \delta(\mathrm{mm} / \mathrm{s}) & \Delta E_{Q}(\mathrm{~mm} / \mathrm{s}) & \eta \\ \text { A1 } & 0.39 & -0.69 & 1 \\ \text { A2 } & 0.48 & -0.94 & 1 \\ \text { A3 } & 0.39 & -0.56 & 1 \\ \text { A4 } & 0.41 & 0.68 & 1 \\ \text { B1 } & 0.33 & -0.66 & 0.9 \\ \text { B1 } & 0.33 & -0.66 & 0.9 \\ \text { B2 } & 0.50 & -0.65 & 1 \\ \text { Mean } & 0.40 & - & -\end{array}$

\section{Results and discussion}

\section{Broken-symmetry states of FeMoco}

The well-known BS-7 solution of FeMoco has been shown by many groups ${ }^{19,34-37}$ to be the energetically most stable according to DFT calculations (maximizing antiferromagnetic 
coupling appears to be the main reason for its stability). However, it is rarely discussed that there are three equivalent BS determinants of nearly the same energy for this state that are obtained by flipping the spins of equivalent iron atoms in the structure (resulting in different spin alignment in the asymmetric protein environment). We refer to these three different solutions as "spin-isomers", as previously discussed by Noodleman et al. ${ }^{34,38,39}$, and label them BS7-235, BS7-346 and BS7-247 with the last 3 numbers referring to the Fe atom numbers of the X-ray crystal structure with "down" spin. While the energy differences between these spin isomers are predicted to be small at the TPSSh level $(\approx 1 \mathrm{kcal} / \mathrm{mol}$ for both crystal structure and for optimized structures; we note that this energy difference is probably functional dependent) and the electronic structure is essentially the same; this also means that $\mathrm{Fe}(2.5)-\mathrm{Fe}(2.5)$ delocalized pairs (that are ferromagnetically aligned) will be on different atoms for the different spin isomers. The delocalized pairs as well as local $\mathrm{Fe}(\mathrm{II})$ and $\mathrm{Fe}(\mathrm{III})$ sites will obviously be sensitive to the geometry used (particularly FeFe distances). Hence when doing single-point property calculations on the X-ray crystal structure, it becomes necessary to consider all 3 spin isomers. The structure of FeMoco from the crystal structure could correspond to the electronic state that is approximately described by one of these BS determinants but could possibly also represent the average of all 3 of them. It remains also to be seen how a multiconfigurational wavefunction description of FeMoco differs from a broken-symmetry DFT description.

Accounting for these broken-symmetry spin isomers is not as crucial, however, when employing an optimized structure as the structure has then relaxed with respect to the spin alignment of the chosen BS determinant. The optimized structures in the next sections all used the BS7-235 solution (TPSSh-optimized) while we considered all 3 spin isomer solutions for the calculations on the crystal structure (see Results section 3.4). Figure S1 in the supporting material shows a geometric comparison of the crystal structure and the BS7-235 TPSSh-optimized structure. 


\section{The effect of calibration on computed Mössbauer isomer shifts}

The magnetic Mössbauer study by Münck et al. ${ }^{17}$ was able to determine individual isomer shifts, quadrupole splitting parameters, quadrupole asymmetry parameters and hyperfine couplings of the Fe atoms in FeMoco. These data are shown in Table 1 (note that the table includes Fe site labels by Münck et al. that are not related to the crystal structure Fe site numbering in Figure 1). Herein we focus only on the average isomer shifts, as the quadrupole splitting parameters for each Fe atom in FeMoco are overall very similar. In addition, the fact that the calculated quadrupole splittings typically have larger errors than the calculated isomer shifts, means that the quantitative insight that can be obtained is more limited.

Computing the Mössbauer isomer shifts for each charge model by quantum chemistry should allow one to distinguish between charge models if the isomer shifts can be accurately determined. ${ }^{57} \mathrm{Fe}$ Mössbauer isomer shifts are typically determined computationally by computing electron density values at Fe nuclear positions (usually by DFT) and converting those values to isomer shifts by using a calibration of experimental isomer shifts that have been correlated with DFT-computed electron densities at the Fe nuclei. Such a calibration was performed by Römelt et al. ${ }^{40}$ for different density functionals and basis sets for a test set of Fe complexes spanning a wide range of oxidation states and ligand environments. When employing such a calibration for computing the Mössbauer isomer shifts of typical iron-sulfur clusters, we discovered, however, that the absolute values were always systematically underestimated (by about $0.11 \mathrm{~mm} / \mathrm{s}$ ), suggesting an unfortunate dependence on the calibration. This would seemingly be related to the lack of iron-sulfur complexes in the test set of Römelt et al. In contrast, Szilagyi et al. ${ }^{19}$ used a test set of iron-sulfur compounds to determine their calibration. That choice could on the other hand be criticized on the basis of the isomer shifts of iron-sulfur compounds spanning only a small range.

In order to explore the effects of calibration on computed FeMoco isomer shifts, new calibrations were created, using the compounds from the Römelt study and a test set of 
iron-sulfur compounds compiled from a a study by Noodleman et al. ${ }^{41}$ and Szilagyi et al ${ }^{19}$. The details are provided in the supporting information. The B3LYP functional was used in all calculations of this section, however, the functional dependence of the results is discussed in the next section.

Shown in Table 2 are isomer shifts computed for all 3 FeMoco charges using the same level of theory, but employing calibration parameters from a fit for the complexes in the study by Römelt et al. (Calibration 1), an iron-sulfur complex test set (Calibration 3) and a calibration determined using the combined Römelt + Iron-sulfur test set (Calibration 2). As can be seen in Table 2, we obtain considerably different numbers depending on how the calibration was carried out. The average isomer shift for $\left[\mathrm{MoFe}_{7} \mathrm{~S}_{9} \mathrm{C}\right]^{3-}$ for example, calculated with Calibration 1, is in very good agreement with the experimental average isomer shift. However, utilization of the iron-sulfur calibration instead (Calibration 3) results in a systematic overestimation of $0.13 \mathrm{~mm} / \mathrm{s}$.

Analyzing the behavior of the isomer shift error for different charge models with respect to the calibration may perhaps be used to suggest one charge model over the others. $\left[\mathrm{MoFe}_{7} \mathrm{~S}_{9} \mathrm{C}\right]^{1-}$ is closest to experiment when employing Calibration 2 (the whole test set) and Calibration 3 (biased towards Fe-S compounds) and $\left[\mathrm{MoFe}_{7} \mathrm{~S}_{9} \mathrm{C}\right]^{1+}$ could perhaps be considered unlikely (as no calibration brings it less than $0.07 \mathrm{~mm} / \mathrm{s}$ to the experimental average). This result is different than obtained in the study by Szilagyi et al. where a more oxidized cofactor $\left(\left[\mathrm{MoFe}_{7} \mathrm{~S}_{9} \mathrm{C}\right]^{1+}\right)$ was found to fit best (using an iron-sulfur compound calibration test set). The most likely reason for this discrepancy appears to be that most of the analysis in the Szilagyi et al. study used the experimental crystal structure (from 2002) instead of DFT-optimized structures. The geometry dependence on the Mössbauer properties will be discussed in a later section.

These results serve primarily as a cautionary note for using computed absolute isomer shifts from DFT calculations without paying attention to the calibration used. This strong 
calibration dependence of the results is unfortunate and arises due to the limited size and composition of the training set, but also perhaps due to Mössbauer data from different sources and the lack of an explicit crystal environment in the computations. We suggest future Mössbauer isomer shift calibration and benchmarking studies to employ even larger tests sets than previously have been used, to perhaps account better for environmental effects and to avoid bias towards a class of compounds as much as possible.

Table 2: B3LYP/CP(PPP) computed electron densities, $\rho_{0}$ values, (TPSSh-optimized geometries for each charge state) and Mössbauer isomer shifts (in $\mathrm{mm} / \mathrm{s}$ ) of the 3 charges of FeMoco using 3 different calibrations. Isomer shifts were computed using the equation: $\delta_{i s o}=\alpha\left(\rho_{0}-C\right)+\beta .{ }^{\mathrm{a}}$ Errors are with respect to the experimental average isomer shift of $0.40 \mathrm{~mm} / \mathrm{s}$.

\begin{tabular}{|c|c|c|c|c|c|c|c|c|c|c|c|c|c|c|}
\hline & $\begin{array}{c}{\left[\mathrm{MoFe}_{7} \mathbf{S}_{9} \mathrm{C}\right]^{3-}} \\
\rho_{0}\left(a u^{-3}\right)\end{array}$ & Cal. 1 & $\begin{array}{l}\mathrm{mm} / \mathrm{s} \\
\text { Cal. } 2\end{array}$ & Cal. 3 & $\begin{array}{c}{\left[\mathrm{MoFe}_{7} \mathrm{~S}_{9} \mathrm{C}\right]^{1-}} \\
\rho_{0}\left(a u^{-3}\right)\end{array}$ & Cal. 1 & $\begin{array}{l}\mathrm{mm} / \mathrm{s} \\
\text { Cal. } 2\end{array}$ & Cal. 3 & $\begin{array}{c}{\left[\mathrm{MoFe}_{7} \mathrm{~S}_{9} \mathrm{C}\right]^{1+}} \\
\rho_{0}\left(a u^{-3}\right)\end{array}$ & Cal. 1 & $\begin{array}{l}\mathrm{mm} / \mathrm{s} \\
\text { Cal. } 2\end{array}$ & Cal. 3 & Exp. FeMoco ${ }^{b}$ & $\mathrm{~mm} / \mathrm{s}$ \\
\hline $\mathrm{Fe} 1$ & 23613.90056 & 0.520 & 0.568 & 0.626 & 23614.57741 & 0.404 & 0.451 & 0.509 & 23615.38558 & 0.266 & 0.310 & 0.369 & $\mathrm{~A} 1$ & 0.39 \\
\hline $\mathrm{Fe} 2$ & 23613.90064 & 0.520 & 0.568 & 0.626 & 23615.20999 & 0.296 & 0.341 & 0.399 & 23615.40080 & 0.264 & 0.308 & 0.366 & A2 & 0.0 .48 \\
\hline $\mathrm{Fe} 3$ & 23614.42929 & 0.430 & 0.476 & 0.534 & 23614.82145 & 0.363 & 0.408 & 0.466 & 23615.72273 & 0.209 & 0.252 & 0.310 & A3 & 0.39 \\
\hline $\mathrm{Fe} 4$ & 23614.91683 & 0.346 & 0.392 & 0.450 & 23615.33535 & 0.275 & 0.319 & 0.377 & 23615.87114 & 0.184 & 0.226 & 0.284 & A4 & 0.41 \\
\hline $\mathrm{Fe} 5$ & 23614.14262 & 0.479 & 0.526 & 0.584 & 23615.35038 & 0.272 & 0.316 & 0.375 & 23615.56218 & 0.236 & 0.279 & 0.338 & B1 & 0.33 \\
\hline $\mathrm{Fe} 6$ & 23614.80501 & 0.366 & 0.411 & 0.469 & 23615.21211 & 0.296 & 0.340 & 0.399 & 23615.67083 & 0.218 & 0.261 & 0.319 & B1 & 0.33 \\
\hline $\mathrm{Fe} 7$ & 23614.92498 & 0.345 & 0.390 & 0.448 & 23615.38229 & 0.267 & 0.311 & 0.369 & 23615.82075 & 0.192 & 0.235 & 0.293 & B2 & 0.50 \\
\hline $\operatorname{ean} \delta_{\text {iso }}(\mathrm{mm} / \mathrm{s})$ & & 0.429 & 0.476 & 0.534 & & 0.311 & 0.355 & 0.413 & & 0.224 & 0.267 & 0.326 & & 0.40 \\
\hline Error $(\mathrm{mm} / \mathrm{s})$ & & +0.029 & +0.076 & +0.134 & & -0.089 & -0.045 & +0.013 & & -0.176 & -0.133 & -0.074 & & \\
\hline
\end{tabular}

a) Calibration 1: $\alpha=-0.1706325, \beta=0.33226751$; Calibration $2: \alpha=-0.1738305, \beta=0.37718458 ;$ Calibration $3: \alpha=-0.1731909, \beta=0.43532389 ; \mathrm{C}$ is 23615. b) Experimental Mössbauer isomer shifts (magnetic Mössbauer measurements performed at $4.2 \mathrm{~K}$ with isotope labelled MoFe protein.) from the study by Münck et al. (the A and B labels are from that study).

\section{Computing relative isomer shifts with respect to the $\mathrm{P}$-cluster}

We want to present a more persuasive argument for the FeMoco charge based on the Mössbauer analysis. In fact the goal of our study is not to compute absolute Mössbauer isomer shifts to the highest accuracy but to use the Mössbauer isomer shifts to differentiate between charge models of FeMoco. Varying the calibration used, only has the effect of shifting the systematic errors present in the calculation. This systematic error, however, can be effectively removed by employing an internal reference instead, i.e. focus on the relative isomer shift of FeMoco with respect to some other Fe compound in the protein with known 
charge. For FeMoco, an obvious internal reference, comes to mind, namely the P-cluster. The P-cluster is a $\left[\mathrm{Fe}_{8} \mathrm{~S}_{7}\right]$ cluster that is also present in the MoFe protein and has a similar unusual double-cubane structure. The P-cluster Fe atoms are known to be all-ferrous according to Mossbauer studies, the average isomer shift being $0.65 \mathrm{~mm} / \mathrm{s}$ for Azotobacter vinelandii, with individual components having isomer shifts spanning the range 0.64-0.69 mm/s, strongly suggesting Fe(II) for all iron atoms. ${ }^{6,42}$ Additionally, the recent SpReAD study $^{21}$ shows no significant differences between the Fe K-edges of the 8 Fe atoms in the cluster, strongly suggesting them all to be in the same oxidation state and Fe(II) (based on edge positions). However, we note that the complex nature of the Fe K-edge rising edge energies, and the contributions of valence delocalization are not yet fully understood. ${ }^{43}$

We can thus convert our absolute chemical shifts (calculated with different calibrations) to relative chemical shifts by subtracting the individual isomer shifts from the computed average isomer shift of the P-cluster (employing the same calibration). Now, plotting the results on this new relative isomer shift scale (Figure 2), one sees that the previous strong calibration dependency has effectively been removed. The B3LYP $\Delta \delta_{i s o}$ values for $\left[\mathrm{MoFe}_{7} \mathrm{~S}_{9} \mathrm{C}\right]^{1-}$ are almost exactly the same with an average of $0.233 \mathrm{~mm} / \mathrm{s}, 0.238$ and $0.237 \mathrm{~mm} / \mathrm{s}$ for calibration 1, 2 and 3. The same applies to $\left[\mathrm{MoFe}_{7} \mathrm{~S}_{9} \mathrm{C}\right]^{3-}(0.115,0.117$ and $0.116 \mathrm{~mm} / \mathrm{s})$ and $\left[\mathrm{MoFe}_{7} \mathrm{~S}_{9} \mathrm{C}\right]^{1+}(0.320,0.326$ and 0.324$)$. The average experimental shift for FeMoco on this same scale is $0.24 \mathrm{~mm} / \mathrm{s}$ and thus it becomes immediately clear that $\left[\mathrm{MoFe}_{7} \mathrm{~S}_{9} \mathrm{C}\right]^{1-}$ shows the smallest deviation from experiment with $\left[\mathrm{MoFe}_{7} \mathrm{~S}_{9} \mathrm{C}\right]^{3-}$ and $\left[\mathrm{MoFe}_{7} \mathrm{~S}_{9} \mathrm{C}\right]^{1+}$ being quite far off. Additionally comparing the individual isomer shifts on the relative scale it becomes clear that the range of relative isomer shift values $(0.14 \mathrm{~mm} / \mathrm{s})$ for $\left[\mathrm{MoFe}_{7} \mathrm{~S}_{9} \mathrm{C}\right]^{1-}$ are in good agreement with the experimental range of values $(0.17 \mathrm{~mm} / \mathrm{s})$, while this is not true for $\left[\mathrm{MoFe}_{7} \mathrm{~S}_{9} \mathrm{C}\right]^{1+}(0.08 \mathrm{~mm} / \mathrm{s})$.

We also explored the functional dependency of our results by repeating all calculations at the BP86 level (a pure GGA functional) and the TPSSh level (a $10 \%$ HF exchange hybrid 
functional). The results are shown in Figure 2 as well and reveal that our analysis generally holds regardless of the functional employed.
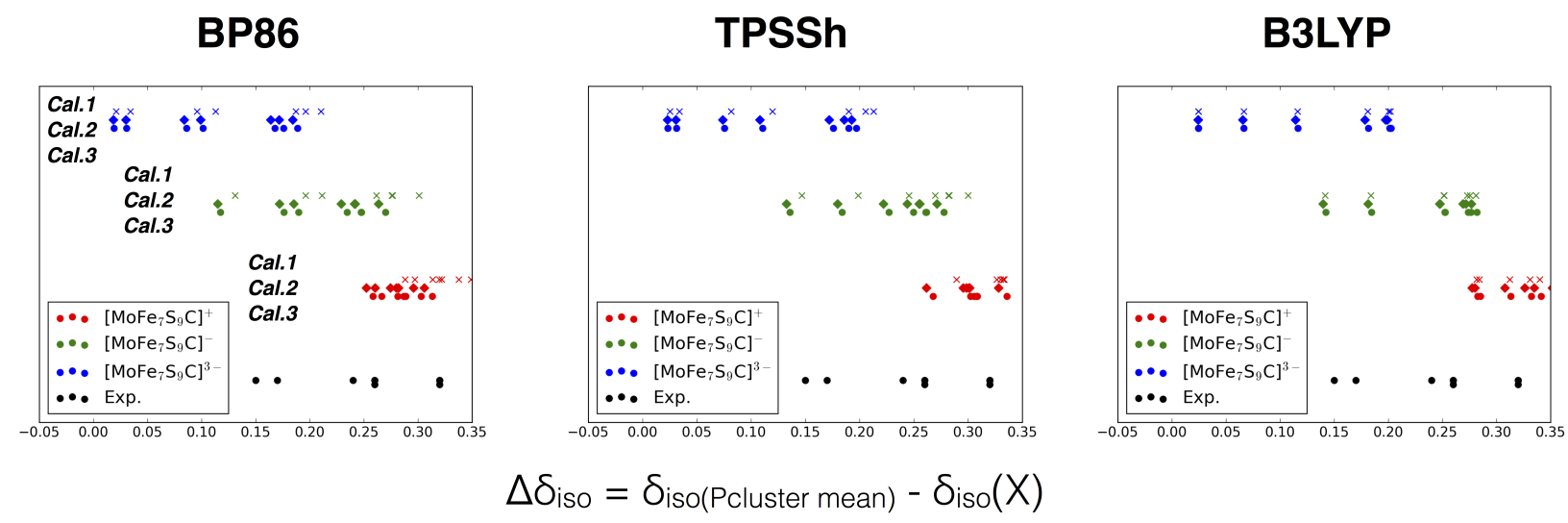

Figure 2: Mössbauer isomer shifts (mm/s) relative to the P-cluster mean using BP86, TPSSh and B3LYP levels of theory on TPSSh-optimized geometries (both FeMoco and P-cluster). For each charge $\left(\left[\mathrm{MoFe}_{7} \mathrm{~S}_{9} \mathrm{C}\right]^{3-}\right.$ shown in blue, $\left[\mathrm{MoFe}_{7} \mathrm{~S}_{9} \mathrm{C}\right]^{1-}$ shown in green and $\left[\mathrm{MoFe}_{7} \mathrm{~S}_{9} \mathrm{C}\right]^{1+}$ shown in red), the values for each calibration are also shown (on the vertical axis, Calibrations 1-3 are shown by crosses, diamonds and circles respectively). Broken-symmetry solution BS7-235 was used in all calculations.

\section{Effect of geometry}

The analysis presented in the previous section relied on the use of DFT geometry optimizations for the FeMoco structures (optimized for each charge state separately), the P-cluster and also all molecules used in the calibration training set. This approach is used in order to minimize artifacts due to static and dynamic disorder present in crystal structures and is the only way of presenting the results on an equal footing. For completeness, however, we also present the Mössbauer results when using the most recent X-ray crystal structure of FeMoco and P-cluster (from the 2011 structure of MoFe protein by Spatzal et al. with 1.0 Ångstrom resolution) for the Mössbauer calculations (all hydrogens positions were optimized). Figure S1 in the supporting materials gives a geometric comparison of the crystal structure and the TPSSh-optimized structure. There are clear similarities between the structures but also 
many notable small differences in the Fe-Fe and particularly Mo-Fe bond lengths.

The Mössbauer isomer shift results for the X-ray structure are presented in Figure 3 and were calculated using Calibration 3 and using all 3 broken-symmetry spin isomers (BS7235,BS7-346,BS7-247), as previously discussed. The results show that the relative isomer shifts can be quite sensitive to the spin alignment but it seems difficult to determine whether the FeMoco crystal structure corresponds to a specific spin isomer.

The $\left[\mathrm{MoFe}_{7} \mathrm{~S}_{9} \mathrm{C}\right]^{3-}$ charge shows considerable deviations from the experimental data and can seemingly be excluded as the charge of the resting state of FeMoco. The $\left[\mathrm{MoFe}_{7} \mathrm{~S}_{9} \mathrm{C}\right]^{1-}$ charge is in good agreement with the experimental shifts for 2 of the spin isomers. The $\left[\mathrm{MoFe}_{7} \mathrm{~S}_{9} \mathrm{C}\right]^{+}$charge is not as sensitive to the spin alignment. Overall, none of the charge models compare as well to the experimental data anymore (both regarding average isomer shift and range of values), and we believe this is due to errors in the metal-ligand (or metalmetal) bond lengths in the X-ray crystal structure adversely affecting the isomer shifts. The previous analysis based on the optimized geometries, however, gave clear and consistent results, strongly indicating the $\left[\mathrm{MoFe}_{7} \mathrm{~S}_{9} \mathrm{C}\right]^{1-}$ charge as the charge of resting state FeMoco.

\section{Interpretation of the electronic structure of $\left[\mathrm{MoFe}_{7} \mathrm{~S}_{9} \mathrm{C}\right]^{1-}$}

With a confident charge assignment of $\left[\mathrm{MoFe}_{7} \mathrm{~S}_{9} \mathrm{C}\right]^{1-}$, we now present an electronic structure interpretation of FeMoco on the basis of the broken-symmetry DFT calculations. We should note from the start that single-reference density functional theory is not a perfect tool for understanding complicated spin-coupled metal clusters. Approximate exchange-correlation functionals have to be used which are known to have limited accuracy for exchange coupling constants, but more severely, electronic states from unrestricted density functional theory are not eigenfunctions of the total spin operator for open-shell molecules. With much progress being made in the last few years in the development and applications of multiconfigurational wavefunction methods that allow for large active spaces, such as the density matrix renor- 

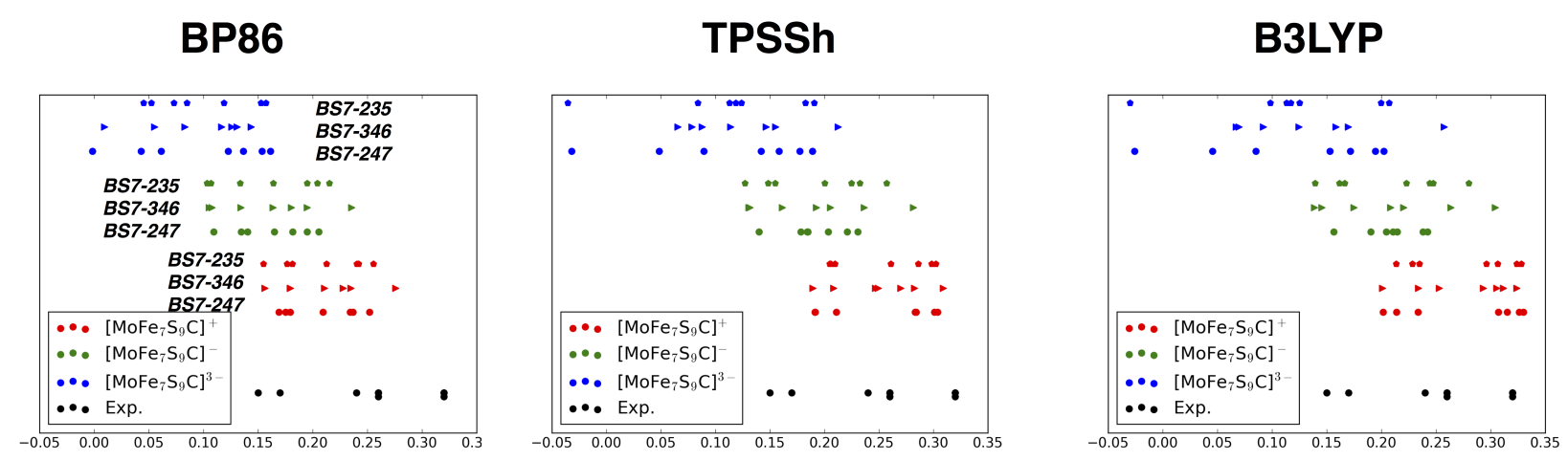

$$
\Delta \delta_{\text {iso }}=\delta_{\text {iso(Pcluster mean })}-\delta_{\text {iso }}(X)
$$

Figure 3: Mössbauer isomer shifts $(\mathrm{mm} / \mathrm{s})$ relative to the P-cluster mean at the BP86, TPSSh and B3LYP levels of theory on the X-ray crystal structure geometry (both FeMoco and Pcluster). For each charge, 3 different spin isomers were calculated; the last 3 numbers in the label refer to which Fe atoms in the model are spin-down (crystal structure numbering). Calibration 3 was used for all these calculations.

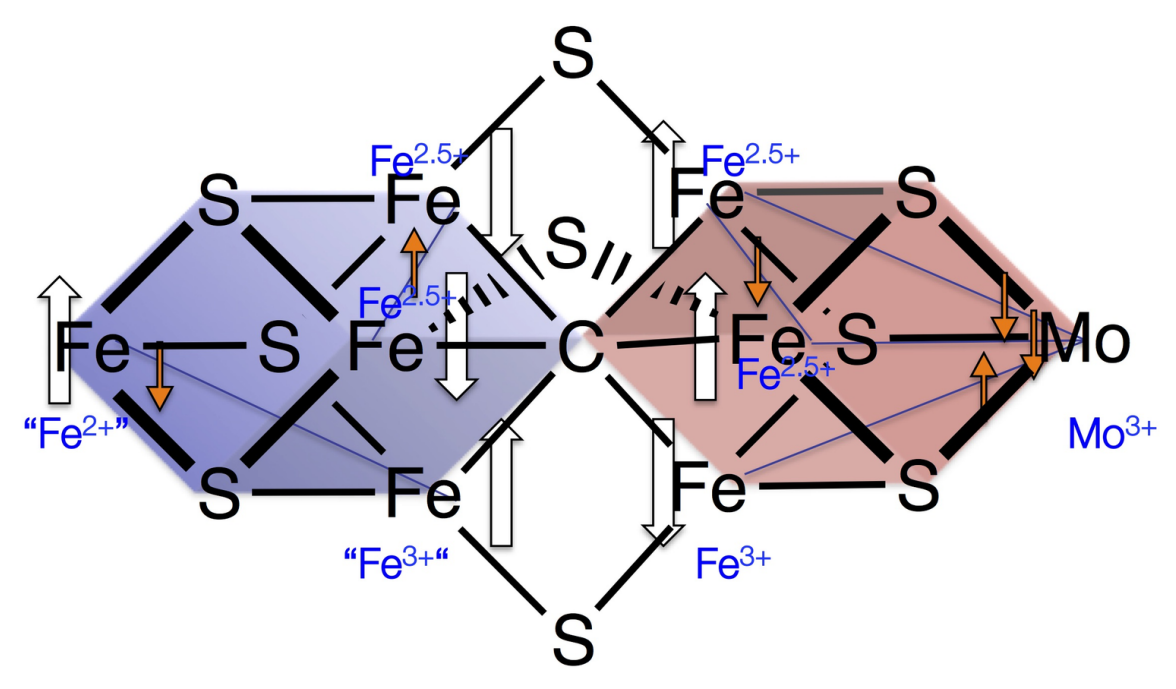

Figure 4: Electronic structure interpretation of $\left[\mathrm{MoFe}_{7} \mathrm{~S}_{9} \mathrm{C}\right]^{1-}$ based on localized orbital analysis. The localized orbitals used to arrive at this diagram are available in the SI.

malization group method (DMRG), ${ }^{44-47}$ we anticipate a more rigourous understanding of the spin coupling of FeMoco to become available in the future. Nonetheless, our DFT calculations on FeMoco can be expected to describe aspects of the electronic structure accurately, such as the charge distribution and valence electronic structure. This is highlighted by the 
previously obtained good agreement between the DFT-optimized FeMoco structures and the calculated XAS/XES spectra. ${ }^{11,48,49}$

Here, we analyze the the electronic structure of FeMoco with the help of localized orbitals that we previously applied in our study of the molybdenum oxidation state in FeMoco. ${ }^{11}$ Localized orbitals are a convenient tool for the analysis of complex electronic structure and are obtained by an appropriate unitary transformation of the canonical orbitals. While not having defined orbital energies anymore, they are just as valid as the canonical orbitals and tend to be easier to understand by simple chemical arguments.

Applying Pipek-Mezey localized orbital analysis ${ }^{33}$ to FeMoco we can gain a qualitative understanding of the spin coupling and delocalization in the broken-symmetry solution found for $\left[\mathrm{MoFe}_{7} \mathrm{~S}_{9} \mathrm{C}\right]^{1-}$. A diagram is shown in Figure 4. The alignment of Fe spins shown, results from the protocol used to find this broken-symmetry solution, where selected Fe atoms are flipped from a ferromagnetic state. This Fe-spin alignment has been referred to as broken-symmetry solution no. 7 by Noodleman and others. This spin alignment maximizes antiferromagnetic coupling in the cofactor as has been discussed previously. ${ }^{19,35}$ As previously discussed, this broken-symmetry solution has 3 spin isomers with analogous electronic structure. In the asymmetric protein environment, subtle differences would arise (both in terms of geometry and electronic structure) and this aspect will be discussed in more detail in a future study. The discussion here (and Figure 4) applies to all spin isomers.

The broken-symmetry solution is typically not discussed in terms of the spin delocalization present (nor has the open-shell nature of the molybdenum been apparent in previous studies, except Refs. ${ }^{11,22}$ ) Three mixed-valence Fe-Fe pairs with parallel spins can be identified. One such pair is in the Mo cubane (i.e. the $\mathrm{MoFe}_{3}$ half of the cofactor) where the spin-down down electron is almost completely shared between the 2 "up-spin" Fe atoms, resulting in an almost completely delocalized $\mathrm{Fe}(2.5)-\mathrm{Fe}(2.5)$ pair. Opposite this pair (in the Fe cubane or the $\mathrm{Fe}_{4}$ half of the cofactor) is another delocalized pair (but with anti-parallel 
spin with respect to pair in the Mo cubane). Finally, the third mixed-valence pair between the capping Fe (labelled Fe1 in the X-ray structure) and the remaining Fe in the Fe cubane, is curiously not as delocalized as the other delocalized pairs; suggesting the capping iron to be have slightly more $\mathrm{Fe}(\mathrm{II})$ character and its partner more Fe(III) character, than the other pairs. This would seemingly be related to breaking of symmetry as the capping Fe and the other Fe are in very different environments (carbide ligand vs. thiolate ligand). Localization of the spin-down electron on the capping Fe would also result in stronger inter-cubane antiferromagnetic coupling between two Fe(III) sites in Fe-cubane and Mo-cubane. The localized orbital analysis is shown in Figure S2 in the supporting material that also shows the amount of delocalization in the pairs for BP86, TPSSh and B3LYP methods.

The delocalization present in the cluster according to our DFT calculations appears at first to be at odds with the recent SpReAD measurements that show three of the Fe atoms in the cofactor (Fe1, $\mathrm{Fe} 3$ and $\mathrm{Fe} 7$ ) to be more reduced than the others and having similar absorption edges as the P-cluster, implying Fe(II) oxidation states. We note that the complex nature of the Fe K-edge rising edge energies, and the contributions of valence delocalization are not fully understood as discussed in a recent study. ${ }^{43}$

Further, we note that the interpretation of the SpReAD data can be rationalized in view of our DFT model if the mixed-valence delocalized pairs are for some reason not completely delocalized. The delocalized pairs in FeMoco as mentioned are not as delocalized (compared to $\mathrm{Fe}_{4} \mathrm{~S}_{4}$ and $\mathrm{MoFe}_{3} \mathrm{~S}_{4}$ cubanes for example) and further localization of the minority-spin electron could be imagined to occur due to symmetry breaking caused by the protein environment (that is probably not described perfectly by our cluster model) or possibly vibronic coupling that can result in localization of mixed-valence Fe(II)-Fe(III) pairs. We also note that localized Fe(II) and Fe(III) sites in FeMoco have also recently been suggested by interpretation of Fe XAS K-edges ${ }^{43}$.

Finally, the diagram shows the Mo atom in the unusual $\mathrm{d}^{3}$ non-Hund configuration that 
has previously been described but is not yet completely understood. This configuration appears to arise due to weak Mo-Fe bonds and the spin alignment of the 3 Fe atoms in the Mo cubane. This unusual configuration also shows up in calculations of synthetic $\left[\mathrm{MoFe}_{3} \mathrm{~S}_{4}\right]^{3+}$ cubanes as well.

\section{Conclusions}

We have presented new DFT calculations of Mössbauer isomer shifts of a large active site model of FeMoco for 3 different charges, all of which have been suggested as possibilities for the resting state charge in the literature. By focusing on computing relative isomer shifts with respect to an internal reference (the P-cluster) instead of the absolute isomer shifts (that depend on careful calibration) we show that only the charge $\left[\mathrm{MoFe}_{7} \mathrm{~S}_{9} \mathrm{C}\right]^{1-}$ is consistent with the experimental Mössbauer isomer shifts. These results thus provide an important starting point for all mechanistic studies of nitrogenase, by clearly establishing the charge of the E0 resting state. We additionally have presented a qualitative interpretation of the electronic structure of this charge of FeMoco based on localized orbital analysis.

\section{Acknowledgments}

We thank Eckhard Bill for valuable discussions and comments on the manuscript. SD and FN acknowledge the Max Planck Society for funding. This work was supported by the European Research Council (ERC) under the European Union's Seventh Framework Programme (FP/2007-2013) ERC Grant Agreement number 615414 (SD). RB acknowledges support from the Icelandic Research Fund, Grant No. 141218051 and the University of Iceland Research Fund. 


\section{References}

(1) Lancaster, K. M.; Roemelt, M.; Ettenhuber, P.; Hu, Y.; Ribbe, M. W.; Neese, F.; Bergmann, U.; DeBeer, S. X-ray Emission Spectroscopy Evidences a Central Carbon in the Nitrogenase Iron-Molybdenum Cofactor. Science 2011, 334, 974-977.

(2) Spatzal, T.; Aksoyoglu, M.; Zhang, L.; Andrade, S. L. A.; Schleicher, E.; Weber, S.; Rees, D. C.; Einsle, O. Evidence for Interstitial Carbon in Nitrogenase FeMo Cofactor. Science 2011, 334, 940-940.

(3) Yang, S.; Pan, W.; Friesen, G.; Burgess, B.; Corbin, J.; Stiefel, E.; Newton, W. Ironmolybdenum cofactor from nitrogenase. Modified extraction methods as probes for composition. J. Biol. Chem. 1982, 257, 8042-8.

(4) Wink, D. A.; McLean, P. A.; Hickman, A. B.; Orme-Johnson, W. H. A new method for extraction of iron-molybdenum cofactor (FeMoco) from nitrogenase adsorbed to DEAEcellulose. 2. Solubilization of FeMoco in a wide range of organic solvents. Biochemistry 1989, 28, 9407-9412.

(5) Huang, H.; Kofford, M.; Simpson, F.; Watt, G. Purification composition, charge, and molecular weight of the FeMo cofactor from azotobacter vinelandii nitrogenase. J. Inorg. Biochem. 1993, 52, 59-75.

(6) Münck, E.; Rhodes, H.; Orme-Johnson, W.; Davis, L.; Brill, W.; Shah, V. Nitrogenase. VIII. Mössbauer and EPR spectroscopy. The MoFe protein component from Azotobacter vinelandii OP. Biochim. Biophys. Acta 1975, 400, 32-53.

(7) Huynh, B.; Henzl, M.; Christner, J.; Zimmermann, R.; Orme-Johnson, W.; Münck, E. Nitrogenase XII. Mössbauer studies of the MoFe protein from Clostridium pasteurianum W5. Biochim. Biophys. Acta (BBA) - Protein Structure 1980, 623, 124-138. 
(8) Huynh, B.; Münck, E.; Orme-Johnson, W. Nitrogenase XI: Mössbauer studies on the cofactor centers of the MoFe protein from Azotobacter vinelandii OP. Biochim. Biophys. Acta (BBA) - Protein Structure 1979, 576, 192-203.

(9) Kim, J.; Rees, D. Structural models for the metal centers in the nitrogenase molybdenum-iron protein. Science 1992, 257, 1677-1682.

(10) Einsle, O.; Tezcan, F.; Andrade, S.; Schmid, B.; Yoshida, M.; Howard, J.; Rees, D. Nitrogenase MoFe-protein at 1.16 A resolution: a central ligand in the FeMo-cofactor. Science 2002, 297, 1696-700.

(11) Bjornsson, R.; Lima, F. A.; Spatzal, T.; Weyhermüller, T.; Glatzel, P.; Bill, E.; Einsle, O.; Neese, F.; DeBeer, S. Identification of a spin-coupled Mo( iii ) in the nitrogenase iron-molybdenum cofactor. Chem. Sci. 2014, 5, 3096-3103.

(12) Hoffman, B. M.; Roberts, J. E.; Orme-Johnson, W. H. Molybdenum-95 and proton ENDOR spectroscopy of the nitrogenase molybdenum-iron protein. J. Am. Chem. Soc. 1982, 104, 860-862.

(13) Venters, R. A.; Nelson, M. J.; McLean, P. A.; True, A. E.; Levy, M. A.; Hoffman, B. M.; Orme-Johnson, W. H. ENDOR of the resting state of nitrogenase molybdenum-iron proteins from Azotobacter vinelandii Klebsiella pneumoniae, and Clostridium pasteurianum. Proton, iron-57, molybdenum-95, and sulfur-33 studies. J. Am. Chem. Soc. 1986, 108, 3487-3498.

(14) True, A. E.; McLean, P.; Nelson, M. J.; Orme-Johnson, W. H.; Hoffman, B. M. Comparison of wild-type and nifV mutant molybdenum-iron proteins of nitrogenase from Klebsiella pneumoniae by ENDOR spectroscopy. J. Am. Chem. Soc. 1990, 112, 651657. 
(15) Burgess, B. K. The iron-molybdenum cofactor of nitrogenase. Chemical Reviews 1990, 90, 1377-1406.

(16) Lee, H.-I.; Hales, B. J.; Hoffman, B. M. Metal-Ion Valencies of the FeMo Cofactor in CO-Inhibited and Resting State Nitrogenase by 57 Fe Q-Band ENDOR. J. Am. Chem. Soc. 1997, 119, 11395-11400.

(17) Yoo, S. J.; Angove, H. C.; Papaefthymiou, V.; Burgess, B. K.; Münck, E. Mössbauer Study of the MoFe Protein of Nitrogenase from Azotobacter vinelandii Using Selective 57 Fe Enrichment of the M-Centers. J. Am. Chem. Soc. 2000, 122, 4926-4936.

(18) Vrajmasu, V.; Münck, E.; Bominaar, E. L. Density Functional Study of the Electric Hyperfine Interactions and the Redox-Structural Correlations in the Cofactor of Nitrogenase. Analysis of General Trends in 57 Fe Isomer Shifts. Inorg. Chem. 2003, 42, $5974-5988$.

(19) Harris, T. V.; Szilagyi, R. K. Comparative Assessment of the Composition and Charge State of Nitrogenase FeMo-Cofactor. Inorg. Chem. 2011, 50, 4811-4824.

(20) Kowalska, J.; DeBeer, S. The role of X-ray spectroscopy in understanding the geometric and electronic structure of nitrogenase. Biochimica et Biophysica Acta (BBA) Molecular Cell Research 2015, 1853, 1406-1415.

(21) Spatzal, T.; Schlesier, J.; Burger, E.; Sippel, D.; Zhang, L.; Andrade, S.; Rees, D.; Einsle, O. Nitrogenase FeMoco investigated by spatially resolved anomalous dispersion refinement. Nat. Commun. 2016, 7, 10902.

(22) Siegbahn, P. E. M. Model Calculations Suggest that the Central Carbon in the FeMoCofactor of Nitrogenase Becomes Protonated in the Process of Nitrogen Fixation. J. Am. Chem. Soc. 2016, 138, 10485-10495. 
(23) Neese, F. The ORCA program system. WIREs Comput. Mol. Sci. 2011, 2, 73-78.

(24) Tao, J.; Perdew, J. P.; Staroverov, V. N.; Scuseria, G. E. Climbing the Density Functional Ladder: Nonempirical Meta-Generalized Gradient Approximation Designed for Molecules and Solids. Phys. Rev. Lett. 2003, 91.

(25) Neese, F.; Wennmohs, F.; Hansen, A.; Becker, U. Efficient approximate and parallel Hartree-Fock and hybrid DFT calculations. A 'chain-of-spheres' algorithm for the Hartree-Fock exchange. Chem. Phys. 2009, 356, 98-109.

(26) Izsak, R.; Neese, F. An overlap fitted chain of spheres exchange method. J. Chem. Phys. 2011, 135, 144105.

(27) van Wüllen, C. On the use of common effective core potentials in density functional calculations. I. Test calculations on transition-metal carbonyls. Int. J. Quantum Chem. 1996, $58,147-152$.

(28) Weigend, F.; Ahlrichs, R. Balanced basis sets of split valence triple zeta valence and quadruple zeta valence quality for $\mathrm{H}$ to Rn: Design and assessment of accuracy. Phys. Chem. Chem. Phys. 2005, 7, 3297.

(29) Pantazis, D. A.; Chen, X.-Y.; Landis, C. R.; Neese, F. All-Electron Scalar Relativistic Basis Sets for Third-Row Transition Metal Atoms. J. Chem. Theory Comput. 2008, 4, 908-919.

(30) Klamt, A.; Schüürmann, G. COSMO: a new approach to dielectric screening in solvents with explicit expressions for the screening energy and its gradient. J. Chem. Soc. Perkin Trans. 2 1993, 799-805.

(31) Neese, F. Prediction and interpretation of the 57Fe isomer shift in Mössbauer spectra by density functional theory. Inorg. Chim. Acta 2002, 337, 181-192. 
(32) Sinnecker, S.; Slep, L. D.; Bill, E.; Neese, F. Performance of Nonrelativistic and QuasiRelativistic Hybrid DFT for the Prediction of Electric and Magnetic Hyperfine Parameters in 57 Fe Mössbauer Spectra. Inorg. Chem. 2005, 44, 2245-2254.

(33) Pipek, J.; Mezey, P. G. A fast intrinsic localization procedure applicable for ab initio and semiempirical linear combination of atomic orbital wave functions. J. Chem. Phys. 1989, 90, 4916.

(34) Lovell, T.; Torres, R. A.; Han, W.-G.; Liu, T.; Case, D. A.; Noodleman, L. Metal Substitution in the Active Site of Nitrogenase MFe7S9(M = Mo4 V3, Fe3). Inorganic Chemistry 2002, 41, 5744-5753.

(35) Lukoyanov, D.; Pelmenschikov, V.; Maeser, N.; Laryukhin, M.; Yang, T. C.; Noodleman, L.; Dean, D. R.; Case, D. A.; Seefeldt, L. C.; Hoffman, B. M. Testing if the Interstitial Atom X , of the Nitrogenase Molybdenum-Iron Cofactor Is N or C: ENDOR ESEEM and DFT Studies of the $\mathrm{S}=3 / 2$ Resting State in Multiple Environments. Inorg. Chem. 2007, 46, 11437-11449.

(36) Kästner, J.; Blöchl, P. E. Ammonia Production at the FeMo Cofactor of Nitrogenase: Results from Density Functional Theory. Journal of the American Chemical Society 2007, 129, 2998-3006.

(37) Dance, I. Electronic Dimensions of FeMo-co the Active Site of Nitrogenase, and Its Catalytic Intermediates. Inorganic Chemistry 2011, 50, 178-192.

(38) Lovell, T.; Li, J.; Liu, T.; Case, D. A.; Noodleman, L. FeMo Cofactor of Nitrogenase: A Density Functional Study of States MN MOX MR and MI. Journal of the American Chemical Society 2001, 123, 12392-12410.

(39) Lovell, T.; Li, J.; Case, D. A.; Noodleman, L. FeMo cofactor of nitrogenase: energet- 
ics and local interactions in the protein environment. Journal of Biological Inorganic Chemistry 2002, 7, 735-749.

(40) Römelt, M.; Ye, S.; Neese, F. Calibration of modern density functional theory methods for the prediction of 57Fe Mössbauer isomer shifts: meta-GGA and double-hybrid functionals. Inorg. Chem. 2009, 48, 784-5.

(41) Sandala, G. M.; Hopmann, K. H.; Ghosh, A.; Noodleman, L. Calibration of DFT Functionals for the Prediction of 57 Fe Mössbauer Spectral Parameters in Iron-Nitrosyl and Iron-Sulfur Complexes: Accurate Geometries Prove Essential. J. Chem. Theory Comput. 2011, 7, 3232-3247.

(42) Huynh, B.; Münck, E.; Orme-Johnson, W. Nitrogenase XI: Mössbauer studies on the cofactor centers of the MoFe protein from Azotobacter vinelandii OP. Biochim Biophys Acta 1979, 576, 192-203.

(43) Kowalska, J. K.; Hahn, A. W.; Albers, A.; Schiewer, C. E.; Bjornsson, R.; Lima, F. A.; Meyer, F.; DeBeer, S. X-ray Absorption and Emission Spectroscopic Studies of [L2Fe2S2]nModel Complexes: Implications for the Experimental Evaluation of Redox States in Iron-Sulfur Clusters. Inorg. Chem. 2016, 55, 4485-4497.

(44) Chan, G. K.-L.; Sharma, S. The Density Matrix Renormalization Group in Quantum Chemistry. Ann. Rev. Phys. Chem. 2011, 62, 465-481.

(45) Yanai, T.; Kurashige, Y.; Mizukami, W.; Chalupský, J.; Lan, T. N.; Saitow, M. Density matrix renormalization group for ab initio Calculations and associated dynamic correlation methods: A review of theory and applications. Int. J. Quantum Chem. 2014, $115,283-299$.

(46) Sharma, S.; Sivalingam, K.; Neese, F.; Chan, G. K.-L. Low-energy spectrum of 
iron-sulfur clusters directly from many-particle quantum mechanics. Nat. Chem. 2014, 6, 927-933.

(47) Kurashige, Y.; Chan, G. K.-L.; Yanai, T. Entangled quantum electronic wavefunctions of the Mn4CaO5 cluster in photosystem II. Nat. Chem. 2013, 5, 660-666.

(48) Bjornsson, R.; Delgado-Jaime, M. U.; Lima, F. A.; Sippel, D.; Schlesier, J.; Weyhermüller, T.; Einsle, O.; Neese, F.; DeBeer, S. Molybdenum L-Edge XAS Spectra of MoFe Nitrogenase. Z. Anorg. Allg. Chem. 2014, 641, 65-71.

(49) Rees, J. A.; Bjornsson, R.; Schlesier, J.; Sippel, D.; Einsle, O.; DeBeer, S. The FeV Cofactor of Vanadium Nitrogenase Contains an Interstitial Carbon Atom. Angew. Chem. Int. Ed. 2015, 54, 13249-13252. 


\section{Synopsis}

The charge assignment of the FeMo cofactor (FeMoco) of molybdenum-dependent nitrogenase has been unclear in the literature. By computing the isomer shifts by density functional theory for each charge and comparing to experiment, we were able to confidently assign the charge of the cofactor as $\left[\mathrm{MoFe}_{7} \mathrm{~S}_{9} \mathrm{C}\right]^{1-}$. Crucial to this work was to compute isomer shifts relative to the P-cluster (another iron-sulfur cluster in the protein) to reduce the systematic errors due to calibration.

\section{TOC figure}

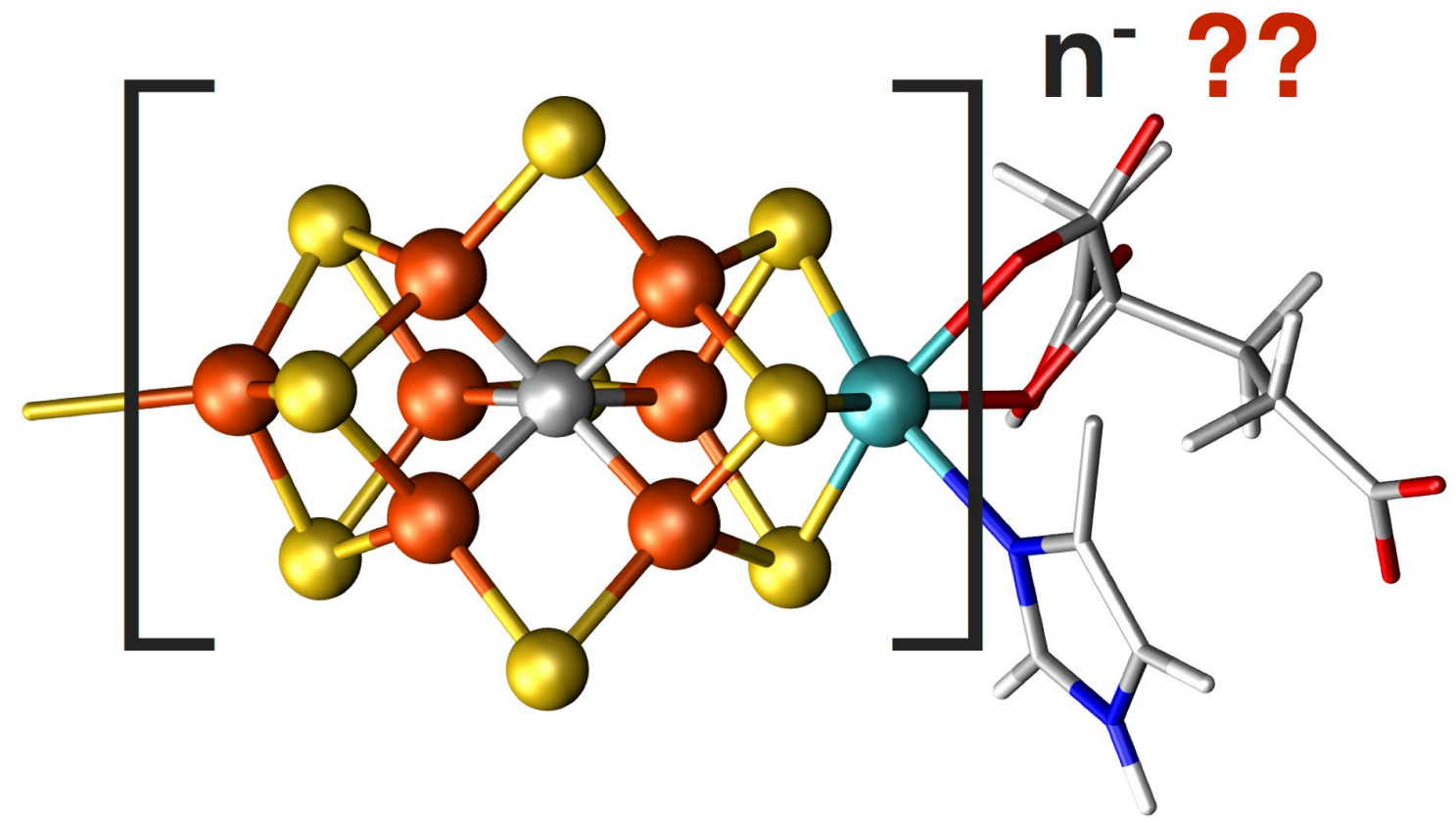

Isomer shifts from DFT

$\left[\mathrm{MoFe}_{7} \mathrm{~S}_{9} \mathrm{C}\right]^{3-}$

$\left[\mathrm{MoFe}_{7} \mathrm{~S}_{9} \mathrm{C}\right]^{1-}$

$\left[\mathrm{MoFe}_{7} \mathrm{~S}_{9} \mathrm{C}\right]^{1+}$
Isomer shifts from

${ }^{57}$ Fe Mössbauer 